\title{
Tumor suppressor p53 stole the AKT in hypoxia
}

\author{
Zhong Yun ${ }^{1}$ and Peter M. Glazer ${ }^{1,2}$ \\ 'Department of Therapeutic Radiology and 'Department of Genetics, Yale University School of Medicine, New Haven, Connecticut, USA.
}

\begin{abstract}
The presence of hypoxia within a tumor is associated with poor clinical outcome, which is often exacerbated by loss of the tumor suppressor $\mathrm{p} 53$. In the presence of functional $\mathrm{p} 53$, hypoxic conditions promote apoptosis; however, the $\mathrm{p} 53$-dependent genes that mediate this process are not well understood. In this issue of the $J C I$, Leszczynska and colleagues identify a p53-dependent six-gene signature that is specifically induced in hypoxia and mediates apoptosis. In patients with a variety of cancers, downregulation of this gene signature was associated with poor clinical outcome. Induction of p53-dependent apoptosis under hypoxia was mediated by AKT inhibition, and treatment with the combination of an AKT inhibitor and ionizing radiation decreased tumor size in a p53-deficient xenograft model more substantially than either single-agent treatment. The results of this study provide important insight into p53-mediated apoptosis under hypoxia and suggest that AKT inhibition has therapeutic potential for inducing apoptosis in hypoxic, p53-deficient cancers.
\end{abstract}

\section{The relationship among hypoxia, p53, and tumor cell apoptosis}

In almost all solid tumors, there are regions of fluctuating and chronic hypoxia due to abnormal vascularization and insufficient perfusion of oxygenated blood. The extent of tumor hypoxia is often associated with therapy resistance and poor clinical outcomes (1). Under severe hypoxic conditions $\left(<0.1 \% \mathrm{O}_{2}\right.$ or $\left.<0.8 \mathrm{mmHg}\right)$, the tumor suppressor p53 becomes stabilized and phosphorylated in the absence of notable DNA damage (2-5). Seminal work by Graeber et al. demonstrated that oncogenic transformation of cells lacking the tumor suppressor $\mathrm{p} 53$ results in resistance to apoptosis under hypoxic conditions, whereas isogenic p53-competent cells are susceptible to hypoxia-induced apoptosis after oncogenic transformation (6). The p53-dependent proapoptotic genes that are induced by hypoxia remain poorly characterized, and the transcription regulatory activity of p53 under hypoxia remains controversial. In this issue, Lesz- czynska et al. provide new mechanistic insight into the p53-dependent apoptosis induced by severe hypoxia (7). As the gene encoding p53 is mutated in many human tumors, the work by Leszczynska et al. makes a substantial contribution toward our understanding of the role of p53 loss or mutation in clonal survival under stress conditions such as severe tumor hypoxia. The insight they provide also suggests a potential approach to improve the therapy of hypoxic tumors.

First of all, the work of Leszczynska and colleagues identifies a p53-dependent proapoptotic pathway that emerges in response to severe hypoxia that is mechanistically different than the p53 pathway that is induced by DNA damage (7). Specifically, Leszczynska et al. examined the role of the DNA-binding domain (DBD) of p53, especially the lysine acetylation sites (K120 and K164), in hypoxia-induced apoptosis of p53-deficient cells. Acetylation of these 2 sites is essential for DNA damage-induced p53-dependent apoptosis (8). Transfection of cells with

\section{Related Article: p. 2385}

plasmids encoding different p53 constructs under the transcriptional control of a hypoxia-inducible promoter revealed that mutation of these lysine residues to arginine (K120R, K164R, and K120R/ K164R) did not markedly affect apoptosis under severe hypoxia $\left(<0.1 \% \mathrm{O}_{2}\right)$. Instead, disruption of the structure of the p53 DBD (R175H mutation) resulted in strong inhibition of apoptosis under the same conditions. These findings suggest that the DNA-binding function is essential for the p53-dependent apoptosis induced by severe hypoxia but that acetylation of the p53 DBD is extraneous.

\section{A hypoxia-specific, p53- dependent gene profile for apoptosis}

Leszczynska and colleagues also identified a panel of six p53-dependent genes that were transcriptionally induced by severe hypoxia in p53-compentent cells (7). Of these p53-dependent transcripts, most were associated with apoptosis (INPP5D, PHLDA3, SULF2, BTG2, and CYFIP2), while one gene (KANK3) had tumor-suppressor function. In contrast, the classical p53-dependent proapoptosis genes, including $B A X, P U M A, N O X A$, and $B I D$, were not induced by hypoxia in a p53-dependent manner. Using chromatin immunoprecipitation, Leszczynska et al. further showed that p53 is capable of binding to promoter/enhancer regions containing p53 response elements in the INPP5D, PHLDA3, SULF2, CYFIP2, and KANK3 genes under severe hypoxia. Interestingly, the canonical hypoxia-inducible factor HIF-1 $\alpha$ was not involved in the hypoxia-induced expression of the above p53-dependent genes. Previously, Fei et al. reported that hypoxia $\left(<0.1 \% \mathrm{O}_{2}\right)$ induces expression of the proapoptotic gene Bnip3l as the result of a synergistic binding of both HIF- $1 \alpha$ and $p 53$ within the Bnip3l promoter (9). The observations by Leszczynska et al. also stand in contrast to previous reports showing that p53 exhibits transcription repressor activities under hypoxia $(4,10)$. It is likely that the transcription activities 


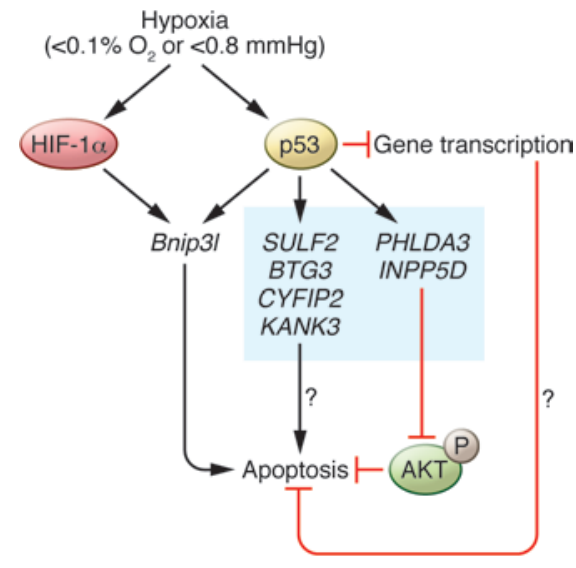

of p53 are potentially regulated by complex mechanisms that are dependent on the target genes, the cell type studied, and the levels of hypoxia (Figure 1).

\section{An AKT-dependent mechanism for p53-depentent apoptosis under hypoxia}

Importantly, Leszczynska and colleagues evaluated gene expression data sets from patients with a variety of cancers and found that an overall suppression of the six hypoxia-induced p53 target genes, but not classical p53-regulated proapoptosis genes, strongly correlates with poor clinical outcomes (7). It will be interesting to further determine whether such a hypoxia-induced p53-dependent gene signature actually has a direct correlation with tumor hypoxia in patients with cancer. Leszczynska et al. further evaluated PHLDA3 and INPP5D, both of which have been previously shown to be capable of promoting apoptosis by inhibiting AKT (11, 12). PHLDA3 encodes a pleckstrin homology domain-containing protein that binds phosphoinositides at the plasma membrane, and INPP5D encodes inositol polyphosphate-5-phosphatase (also known as SHIP-1), which negatively regulates cell proliferation and survival. In WT p53-expressing cells, hypoxia preferentially increased the expression of a short INPP5D transcript from an internal promoter, and overexpression of $P H L D A 3$ in p53-deficient cells notably increased apoptosis in response to hypoxia. Conversely, knockdown of either PHLDA3 or INPP5D substantially improved cell survival in WT cells. Moreover, combined pharmacological SHIP-1 inhibition and PHLDA3 knock-

Figure 1. p53-dependent apoptosis via inhibition of AKT activation under severe hypoxia. Severe hypoxia promotes transcription of the proapoptotic gene Bnip3l, which requires both HIF-1 $\alpha$ and p53 binding at the Bnip3/ promoter. Previous studies have suggested that hypoxia alters the transcriptional activity of $\mathrm{p} 53$ by inducing $\mathrm{p} 53$ to function as a transrepressor as opposed to a transactivator. In this issue, Leszczynska and colleagues identified a six-gene signature (blue box) that promotes apoptosis in p53-expressing cells under hypoxia. The products of two of these genes, PHLDA3 and INPP5D, prevent phosphorylation and activation AKT, thereby promoting apoptosis.

down further blocked hypoxia-induced apoptosis. Both PHLDA3 knockdown and pharmacological inhibition of SHIP-1 led to sustained phosphorylation of AKT at S473 under hypoxia, prompting Leszczynska and colleagues to test the hypothesis that AKT inhibition would enhance apoptosis of p53-deficient cells under hypoxia. As predicted, pharmacologic inhibition of AKT with MK-2206 increased apoptosis of p53-deficient cells and improved tumor growth control in combination with ionizing radiation in murine xenograft models.

AKT activation (as measured by $\mathrm{S} 473$ phosphorylation) has been observed in several tumor types, including breast cancer (13), head and neck cancer (14), and lung cancer (15). Leszczynska and colleagues found sustained AKT-S473 phosphorylation in p53-deficient cells but not in p53-expressing cells under severe hypoxia (7). Furthermore, AKT activation was negatively regulated by the gene products of PHLDA3 and INPP5D in WT p53-producing cells exposed to severe hypoxia, indicating that decreased PHLDA3 and INPP5D expression contributes to sustained AKT phosphorylation in p53-deficient cells under hypoxia. Together, these results indicate that combining AKT inhibitors with genotoxic agents, such as ionizing radiation, can potentially overcome AKT-mediated therapy resistance of hypoxic tumors and thus improve clinical outcomes of patients with cancer.

\section{Conclusions and future directions}

It is worth noting, however, that the median $\mathrm{pO}_{2}$ in several major tumor types has been found to be approximately
$10 \mathrm{mmHg}$ or $1.3 \% \mathrm{O}_{2}$ (16). In addition, tumor hypoxia is often heterogeneously distributed within the solid tumor proper and fluctuates both in duration and degree (17); therefore, the mechanism proposed by Leszczynska et al. is likely to operate primarily in regions of very low oxygenation $\left(<0.1 \% \mathrm{O}_{2}\right.$ or $<0.8$ $\mathrm{mmHg}$ ). Furthermore, hypoxia can both globally and selectively impact gene expression by multiple epigenetic mechanisms (18-20). Such hypoxia-induced epigenetic modifications may potentially interact or interfere with p53-dependent transcription activities and may perhaps alter p53 function. Depending on cellular context and/or the nature of hypoxia, p53 may switch from being a transcriptional activator to functioning as a transcriptional repressor. Nevertheless, AKT inhibitors are in active clinical development (21), and the work reported by Leszczynska et al. points to a novel and potentially important application for such agents, with broad implications for cancer therapy.

\section{Acknowledgments}

This work was funded in part by grants from the NIH: R01CA148996 (Z. Yun and P.M. Glazer), R01CA178254 (Z. Yun), and R01ES005775 (P.M. Glazer).

Address correspondence to: Zhong Yun and Peter Glazer, Department of Therapeutic Radiology, Yale School of Medicine, PO Box 208040, New Haven, Connecticut 06520-8040, USA. Phone: 203.737.2183; E-mail: zhong.yun@yale. edu (Z. Yun). Phone: 203.737.2788; Email: peter.glazer@yale.edu (P.M. Glazer). 
1. Vaupel P, Mayer A. Hypoxia in cancer: significance and impact on clinical outcome. Cancer Metastasis Rev. 2007;26(2):225-239.

2. Hammond EM, Denko NC, Dorie MJ, Abraham RT, Giaccia AJ. Hypoxia links ATR and p53 through replication arrest. Mol Cell Biol. 2002;22(6):1834-1843.

3. Hammond EM, Dorie MJ, Giaccia AJ. ATR/ATM targets are phosphorylated by ATR in response to hypoxia and ATM in response to reoxygenation. J Biol Chem. 2003;278(14):12207-12213.

4. Koumenis C, et al. Regulation of $\mathrm{p} 53$ by hypoxia: dissociation of transcriptional repression and apoptosis from p53-dependent transactivation. Mol Cell Biol. 2001;21(4):1297-1310.

5. Lee JH, et al. Hypoxia activates tumor suppressor $\mathrm{p} 53$ by inducing ATR-Chk1 kinase cascademediated phosphorylation and consequent 14-3-3 $\gamma$ inactivation of MDMX protein. J Biol Chem. 2012;287(25):20898-20903.

6. Graeber TG, et al. Hypoxia-mediated selection of cells with diminished apoptotic potential in solid tumours. Nature. 1996;379(6560):88-91.

7. Leszczynska KB, et al. Hypoxia-induced p53 modulates both apoptosis and radiosensitivity via AKT. J Clin Invest. 2015;125(6):2385-2398.

8. Mellert HS, et al. Deacetylation of the
DNA-binding domain regulates p53-mediated apoptosis. J Biol Chem. 2011;286(6):4264-4270.

9. Fei $\mathrm{P}$, et al. Bnip3L is induced by $\mathrm{p} 53$ under hypoxia, and its knockdown promotes tumor growth. Cancer Cell. 2004;6(6):597-609.

10. Achison M, Hupp TR. Hypoxia attenuates the p53 response to cellular damage. Oncogene. 2003;22(22):3431-3440.

11. Kawase T, et al. PH domain-only protein PHLDA3 is a p53-regulated repressor of Akt. Cell. 2009;136(3):535-550.

12. Liu Q, et al. SHIP is a negative regulator of growth factor receptor-mediated $\mathrm{PKB} / \mathrm{Akt}$ activation and myeloid cell survival. Genes Dev 1999;13(7):786-791.

13. Wennemers M, et al. Hypoxia regulation of phosphokinases and the prognostic value of pAKT in breast cancer. Int J Biol Markers. 2013;28(2):151-160.

14. Stegeman $\mathrm{H}$, et al. Activation of AKT by hypoxia: a potential target for hypoxic tumors of the head and neck. BMC Cancer. 2012;12:463.

15. Lee SM, Lee CT, Kim YW, Han SK, Shim YS, Yoo CG. Hypoxia confers protection against apoptosis via PI3K/Akt and ERK pathways in lung cancer cells. Cancer Lett. 2006;242(2):231-238.
16. Vaupel P, Hockel M, Mayer A. Detection and characterization of tumor hypoxia using pO2 histography. Antioxid Redox Signal. 2007;9(8):1221-1235.

17. Dewhirst MW, Cao Y, Moeller B. Cycling hypoxia and free radicals regulate angiogenesis and radiotherapy response. Nat Rev Cancer. 2008;8(6):425-437.

18. Lu Y, Wajapeyee N, Turker MS, Glazer PM. Silencing of the DNA mismatch repair gene MLH1 induced by hypoxic stress in a pathway dependent on the histone demethylase LSD1. Cell Rep. 2014;8(2):501-513.

19. Lu Y, Chu A, Turker MS, Glazer PM. Hypoxiainduced epigenetic regulation and silencing of the BRCA1 promoter. Mol Cell Biol. 2011;31(16):3339-3350.

20. Krieg AJ, Rankin EB, Chan D, Razorenova O, Fernandez S, Giaccia AJ. Regulation of the histone demethylase JMJD1A by hypoxiainducible factor 1 alpha enhances hypoxic gene expression and tumor growth. Mol Cell Biol. 2010;30(1):344-353.

21. Rodon J, Dienstmann R, Serra V, Tabernero J. Development of PI3K inhibitors: lessons learned from early clinical trials. Nat Rev Clin Oncol. 2013;10(3):143-153. 